\title{
Space Controller Measurement System Based on LabVIEW
}

\author{
Juan Chen ${ }^{1, ~ a, ~ Y o u w e i ~ Z h u^{1, b}, \text { Jian Zhang }}{ }^{1, ~ c}$, Liyang Wang ${ }^{1, d}$ \\ ${ }^{1}$ Beihang University, Beijing 100191, China \\ a 55894833@qq.com, b buaa_zyw@163.com, ${ }^{\mathrm{c}}$ 1226014264@qq.com , d1401079106@qq.com
}

Keywords: Space Controller, Measurement System, LabVIEW, Multi Loops Model of Producer/consumer

Abstract. In order to solve the difficulty in testing space controller, a general measurement system is designed and integrated. Based on LabVIEW, the multi loops model of producer/consumer is chosen to program which makes the interaction and sharing of data came true. Using the structure of state machines, the measurement task is completed by the measurement system efficiently and reliably. Following the MIL-STD-1553B bus protocol, completed 1553B communication test between host computer and space controller. Through the process of the noise reduction, the noise of the experimental data is effectively eliminated or reduced so that the measurement accuracy is improved. The space controller measurement system that is based on LabVIEW makes high-speed data acquisition, real-time processing, synchronous display and parallel control requirements come true, which has generality and extension as well.

\section{Introduction}

Space controller is the core component of aerospace products, more complex than the common controller. It has more types of signals, so as to meet the important role of the controller. This system needs to test not only the traditional digital signal, analog signal, but also requires establishment communications of 1553B bus communication, serial port communication, Ethernet communication between the host computer and the controller, the measurement content are shown in Table.1. With the development of modern technology and modern industrial production, the reliability of aerospace products become more and more important. Measurement content and measurement process is more complex, measurement workload increases, the requirements of speed and precision is also constantly improving [1].

Table.1 measurement content table

\begin{tabular}{|c|c|}
\hline Signal types & The number of channels \\
\hline Sine signal & 40 \\
\hline Modulation signal & 40 \\
\hline Graded DC signal & 80 \\
\hline Digital signal & 128 \\
\hline 1553B bus communication & 2 \\
\hline Serial port communication & 2 \\
\hline Ethernet communication & 2 \\
\hline
\end{tabular}

This system uses the I / 0 interface equipment to complete the signal acquisition, measurement and control, uses powerful software of host computer to realize the signal operation, analysis and processing, to complete all kinds of measurement contents. Measurement software worked in Host computer is programmed in the NI LabVIEW. LabVIEW is the most widely used , fastest-growing, most powerful virtual instrument development software, using data flow diagram makes the program simple and clear, give full play to the advantages of graphical programming environment, shorten the development cycle, eliminate the virtual instrument of virtual instrument programming of complex process. Based on the LabVIEW, the author develops a set of space controller measurement system [2]. 


\section{Composition of measurement system}

Hardware of this controller measurement system consists four main parts of sensors, signal conditioning circuit, data acquisition cards, host computer, as shown in Fig.1. Sensors will be used in signal acquisition controller; Signal conditioning module will regulate voltage signals of the data acquisition collected to the range that data acquisition card can read. Data acquisition card is used to collect all kinds of signals that are adjusted for it. Collected signal is operated, analyzed processed and displayed on the host computer.

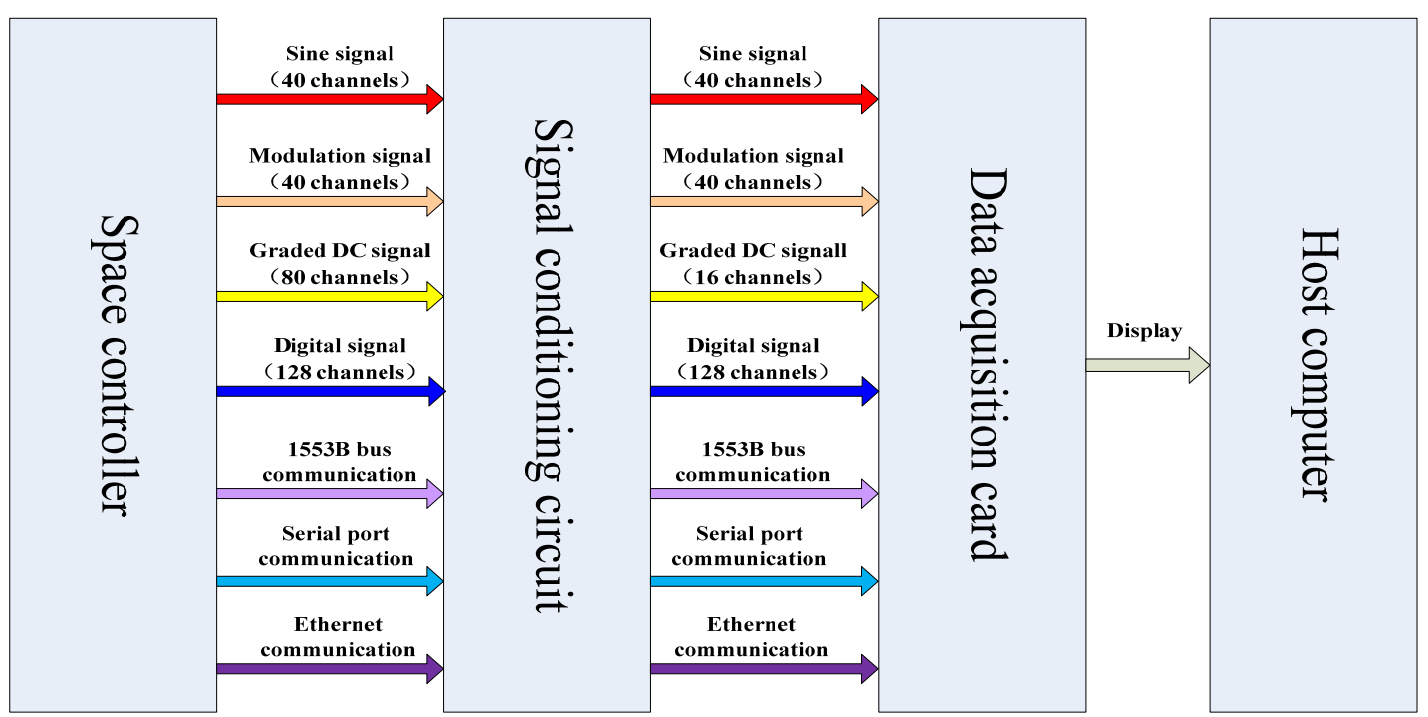

Fig.1 hardware of measurement system

Software of the test system is the key part, the software structure of this system is divided into three parts: input/output (I/O) interface software, instrument driver and development environment of application software. Input/output interface software exists between instrument and instrument driver, is to complete to directly access data instrument internal register unit operation. Instrument driver provides users with more abstract operation function set for instrument operation. Application software is used for programming.

\section{Realization of the core technology}

\subsection{Software architecture}

In this system, multi loops model of producer/consumer is adopted to program to realize the interaction and sharing of data, preventing date overflow or insufficient in data buffer when the amount of data acquisition and obtained is different at the same time. Queue function of LabVIEW provides the good way to circumvent this problem. As the elements in the queue follows the rule of "First in First out", ensured that the data received is ordered [3].

The program has five main loops: the main loop, main program state machine, data acquisition state machine, data save state machine, data display state machine, as shown in Fig.2. Main loop combined of event structure is used to respond to user's interface operation, and will send received commands in the form of a message to other state machines to finish the corresponding task. The main program state machine performs the operation of the system configuration. Data acquisition state machine has the configured channel finish the task of data acquisition. Data save state machine classifies and saves acquired data. Data display state machine responds to user operation and display the requirement data acquired and curve generated. 


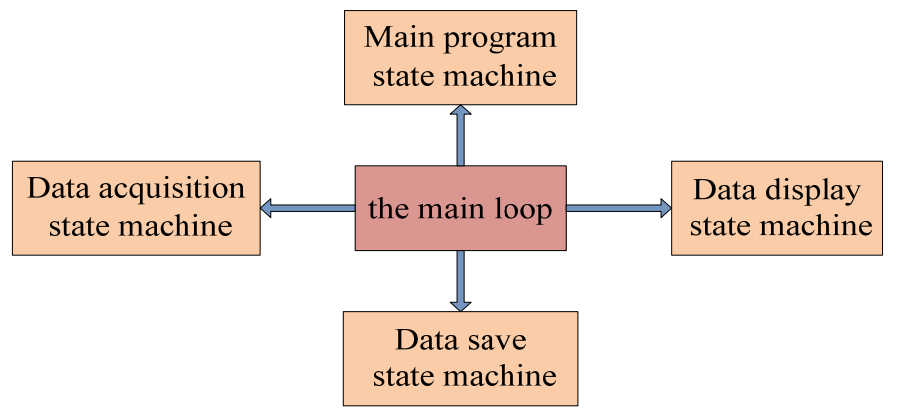

Fig.2 multi loops model of producer/consumer

\subsection{Measurement process}

Measurement process is plays an important role in measurement software. Reasonable testing process makes the software more efficient, better to complete the test task, the measurement process is shown in Fig.3.

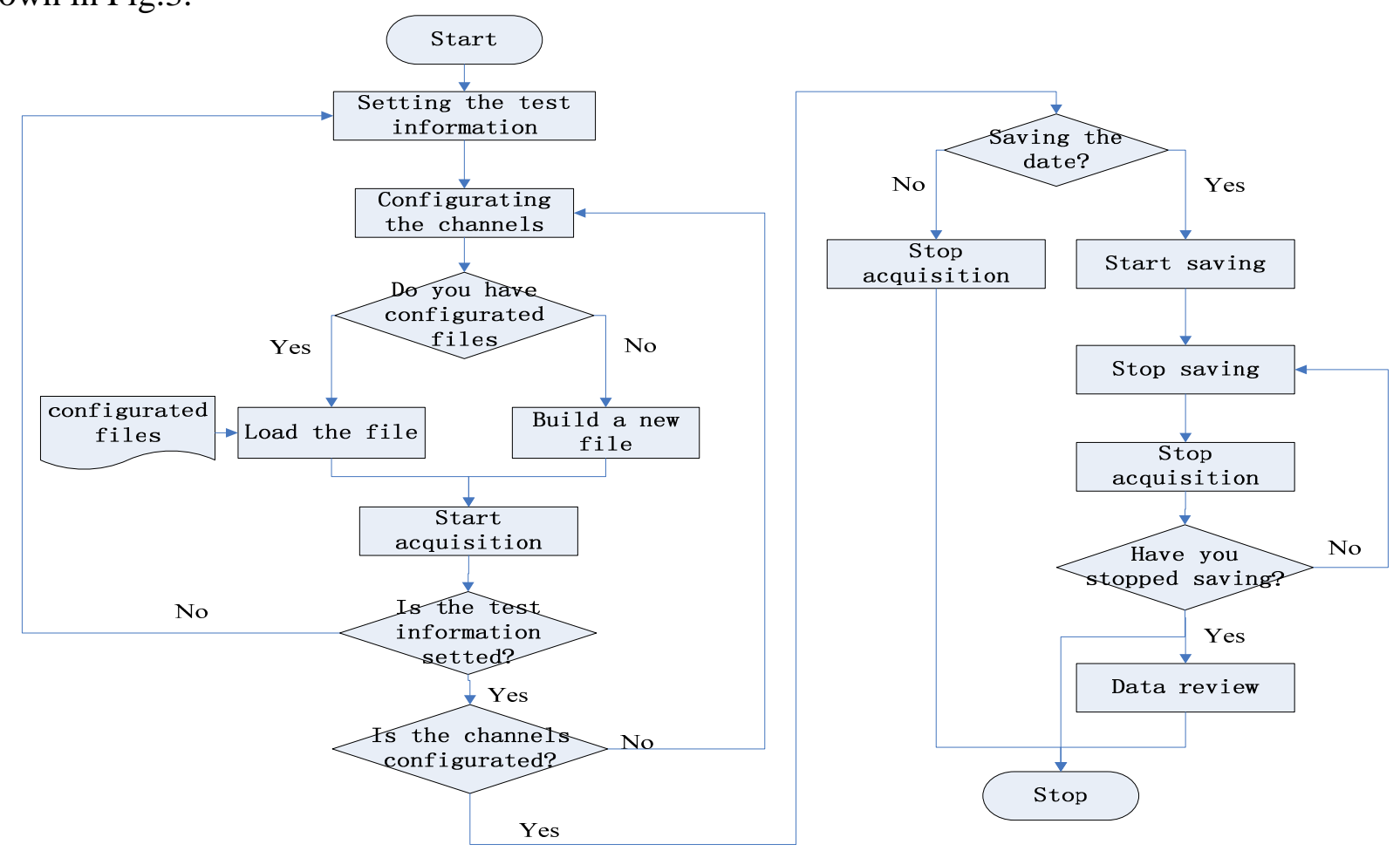

\subsection{B bus communication}

Fig. 3 the measurement process

In order to achieve the task of 1553B bus communication, the host computer can be used as BC (Bus Controller) to control other bus stations, or used as MT (Monitor) to monitor all data on the bus. With the help of card PXI-1553-2D, following the MIL-STD-1553B bus protocol, adopting the Manchester II bipolar encoding format, implements the communication between host computer and controller, the simulation waveform diagram as shown in Fig.4. The 1553B communication test between host computer and controller is completed [4]. 


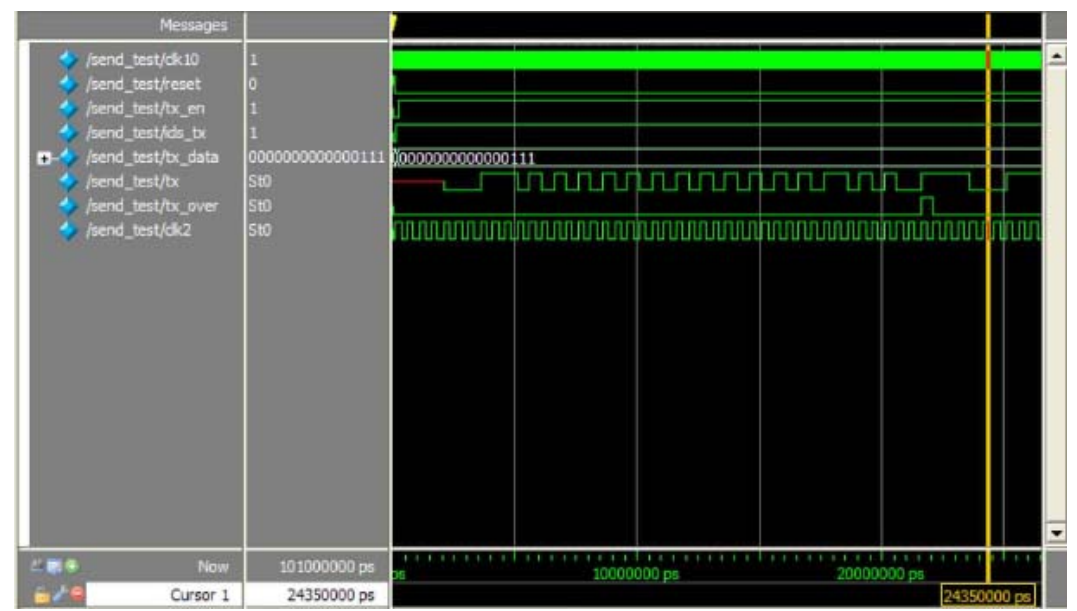

Fig.4 the simulation waveform of 1553B

\section{Noise reduction}

Test precision is important evaluation indexes of measurement system. Noise is the main factor affecting the accuracy in the measurement process. Shielding and twisted-pair wire is used to against noise signal lines into the measurement system. At the same time in software of the host computer, some of the measures are taken to eliminate or reduce the noise [5].

(1) On average. The noise of the sampling of the signals after an average can be roughly decreased to the square root of the average number.

(2) Using analog filter. Analog filter is used to weaken the specific frequency range of the input signal. Analog filter including low-pass filter, high-pass filter, band-pass filter, and band-stop filter.

(3) Eliminating singularity. Using the difference method on the math to detect and eliminate singularities.

\section{Conclusion}

(1) This system adopts the multi loops model of producer/consumer, completes the test task efficiently and accurately.

(2) Completed 1553B communication, the host computer as the BC can control the space controller as a RT, and the host computer as the MT can monitor all data of other bus stations.

(3)Based on LabVIEW, this system can complete space controller or other similar mechanical and electrical products testing with high-speed data acquisition, real-time processing, synchronous display and parallel control.

\section{References}

[1] Wang X. L, Xu H, Dang RP, Communication Test System Based On Networked Inrtument and Virtual Instrument, Information Technology, (2013) , P.63-65

[2] Zhang X. H, Gong W. Q, Liu C. C. Study on Multihole Pressure Probe System Based on LabVIEW, Experimental Techniques, Vol.39 (2015) , P.42-54

[3] Chou J. C, Lin C. Y. Data Fusion and Fault Diagnosis for Flexible Arrayed pH Sensor Measurement System Based on LabVIEW, Vol.14, Sensors Journal,( 2014), P.1405-141

[4] Wang L. The Design of the Monitoring System for the 1553B Bus based on FPGA \& LabVIEW, Chengdu University of Technology(2013)

[5] Chen D. Research on Multi-channel Parallel Dynamic Testing Virtual Instrument Based on LabVIEW, Nanjing University of Aeronautics and Astronautics(2005) 